Volume 9, No.5, September - October 2020

International Journal of Advanced Trends in Computer Science and Engineering

Available Online at http://www.warse.org/IJATCSE/static/pdf/file/ijatcse101952020.pdf

https://doi.org/10.30534/ijatcse/2020/101952020

\title{
Mood Detection using Facial Recognition Technology Application in Higher Education Institution
}

\author{
Taif Alrayassi ${ }^{1}$, Samar Shilbayeh ${ }^{2}$ \\ ${ }^{1}$ Abu Dhabi School of Management, UAE, adsm-213014@adsm.ac.ae \\ ${ }^{2}$ Abu Dhabi of Management,UAE, s.shilbayeh@adsm.ac.ae
}

\begin{abstract}
Mood detection is an important trait that human can do using face expressions and it could be learned by the machine using machine learning approaches and technology. This research utilized a deep learning algorithm to develop a model that can detect various mood of participants in order to identify their subject areas of interest when enrolling in higher education institution so the academic advisor can smartly advise and guide the students in selecting the right program when they are seeking admission to the college.
\end{abstract}

The methodology used in this research is application of the pre trained model technology deployed by convolution neural network for analyzing the facial expression on participants through three steps namely: image processing, facial recognition and mood detection. The mood detection by facial recognition technology is used to test independent input images of 15 participants to detect whether the mood is "happy", "sad", "neutral" and "surprise". The student's mood is determined by the facial movement this can further help in guiding the students for the proper program selection based on their facial expressions. A quasi experimental approach has been taken to analyze the results.

The research findings show that the developed model was able to predict and classify various mood quiet accurately such as "happiness" and "neutral". On the other hand, difficultly detect other moods such as "surprise" and "sad".

The study shows promising results and great capability of detecting human moods that has lots of applications such as academic advising in academic institution. The research is yet a good contribution in identifying a student's facial expression that positively effects the academic advising process and it is expected to better understand the student's needs and emotions.

Key words: Artificial Intelligence, Image Processing, Mood detection, Neural Networks

\section{INTRODUCTION}

Human face is the easiest way to identify human expression and detect his/ her mood. Understanding what other people think and feel can be done easily by analyzing people facial expression. Scientists agree that facial movements convey a range of information and are important for social communication [1]- [3]. Mood detection is one of the most important nonverbal communication during interviews. It provides countless emotions without telling a word. According to [4] different emotional behavior effects decision making process in different ways. If a person is in a state of sadness, certainly there will be more compromises and will settle for things which are not favorable. Similarly, emotions meagerly don't affect the nature of decision but also effects the whole decision-making process in terms of speed of making decisions. Emotion such as anger can lead to impatience and rash decision making whereas excitement can lead quick decision making without taking into account the risk and implications about the decision as there is flow of wave of confidence and optimism for the future. As well as emotions such as fear can lead to longer decision-making process as there will be lot of apprehensions, caution and uncertainty associated with it. Academic advising interview is a very valuable step for students. The advisor helps the students to make decision about the appropriate major selection. Academic advising can play a key role in students' progress toward attaining confidence and achieving their goals. Without the guidance of an academic advisor, the students may find themselves lost and might spent longer time in college because of the wrong choice that they have made in the beginning. On the other hand, the role of advisor is not only limited to a pure academic guidance, but also includes the ability to understand the student's mood in order to maintain a better interactive during the interview. Moreover, the advisor should have a large amount of knowledge about all majors as well as share insights and important statistics based on his/her experiences in order to convince the student about the appropriate major. These are very precise points to ensure that students have enough use of available information to make a better decision regarding to their study plan. However, in practice there are many students are assigned to one advisor, so there is no enough time to assist the students. Some academic advisor has a lack of knowledge about various majors. Thus, the students are frustrating and yield a bad decision of major selection. In some cases, it's hard for some advisor to understand the student mood during the interview which lead to inability to convince the student about the right major as well as inability to motivate him /her to continue the enrollment in the college. 
In this research, a machine learning algorithm is utilized to provide a solution that is able detect the student mood during the admission process. Accordingly, advising interview and the orientation process which will enable the student to make right decision more efficiently. Furthermore, this will help and motivate the student to select the proper major. The solution will be integrated with a camera that capture the student's facial expression then the mood will be analyzed through the movement of certain facial muscles to categorize it into four emotions: "happy", "sad", "neutral" and "surprise". This solution is proposed and intended to be applied in Abu Dhabi School of management (ADSM) for the aim of enhance the process of academic advising.

The focus of this work is to utilize machine learning technology to develop a solution that is capable of detecting the student mood. For the aim of building the right model, the processes composed of 3 stages:

1- Image processing (by selecting multiple features like eyes, eyebrows, mouth and forehead. the ada boost method will be applied)

2- Facial feature extraction (This stage includes dimensionality reduction, feature extraction which can be achieved by applying PCA algorithm)

3- Mood detection (Neural networkwill be deployed because of its effectiveness for image)

\section{Literature ReVIEW}

\subsection{Theoretical Background}

This section presents the literatures found in different machine learning approaches in detecting the human mood. There are various approaches and technology were developed and implemented to classify the facial features across dataset for training and testing. The recent relevant works consist of three stages: image processing, facial features extraction and mood detection (classification).

\subsubsection{Image Processing}

Many researchers have applied neural network to classify human facial expressions, in researches [5, 7] the concepts of committee neural network using which includes several sets of neural network algorithms are applied in the training face for the aim of image classification. It is found in the mentioned researches that committee neural networks outperform a single neural network in detecting and classification each part of the input tested images. Based on these studies, an intelligence system is developed in $[8,26]$ to classify the human's facial expressions by using committee neural network. The developed system was able to classify the input images into seven facial expressions ("No Emotion", "happy", "sad", "disgust", "angry", "fear" and "surprised"). On the other hand, it is suggested in [10] to apply principle component analysis (PCA) as one of three components in the facial expression recognition system, the PCA algorithm is used to gain major trends through hidden patterns in high dimensional data, and for the aim of data reduction.
Experiment in $[10,27]$ is done using Japanese female facial features database. The developed system recognized 6 emotions: "sad", "fear", "surprised", "happy", "angry" and "disgust". The result of this research is $91.63 \%$ accuracy $72.82 \%$ is the precision rate.

\subsubsection{Facial Features Extraction}

Facial features extraction is the process of extracting multiple face elements from human image like eyes, nose, mouth, eyebrow, forehead, etc. This is very important in detecting mood using facial recognition. It includes dimensionality reduction, features extraction and feature selection. According to [11, 24, 25] weighted Principal Component Analysis (WPCA) method is used on multi feature combination. High dimensional features are extracted from facial expression images; as a result, the images are separated into sections. After that WPCA is used in reducing the dimensions; the weights are quickly defined based on facial action coding system, also Radial Basis Function (RBF) is used. At the end, Euclidean distance is computed to gain the similarity between templates and then facial expression recognition is done with nearest algorithm, thus the detection rate is $88.25 \%$ in this method. Alternatively, the researcher in $[11,22,23]$ has proposed a new linear discriminant analysis which is based on facial recognition system. In this paper more efficient, accurate and stable methods will be proposed to compute the discriminant vectors based on Fisher's criterion and two steps processes are implemented. In the first step, the homogeneous areas of a facial images are classified on the same patterns based on geometric characteristics, later the mean gray value is used for pixels within the partition area, consequently the face image is reduced as feature vector. In the second step the feature vector is used to determine discriminant projection axes based on the suggested LDA.

\subsubsection{Mood Detection (Classification)}

This is an important process in facial recognition. Over the decades, researchers in [12] proposed numerous methods to classify human facial features into emotions using facial recognition. In the proposed solution a facial expression recognition and classified into seven emotions: happiness, disgust, fear, No Emotion, anger, sadness and surprise. The system is prepared without characteristic blocks. In this study skin color detection technique is used for face detection that is YCbCr,HSI,RGB color family is applied for coding the images. Then the skin color areas is separated from non-skin color area by means of lower and upper bound threshold, hence the mean value of range of skin colors is taken between 3 and 38. subsequently the facial feature detections are done using Color Space Transformation, Connected Component Labeling Technique, Face area Verification like face segmentation, Pupils detection like referring height and width of the face area etc. Therefore, the landmarks areas like mouth, eyes, eyebrows of the facial features are obtained. Finally, the facial expressions are discriminated by the movement of landmark facial features by the algorithm proposed by the researcher using AdaBoost based classifier. A different method is applied by the researchers in [13, 25, 26]. 
In his study there is combination of two methods Feature Extraction and Neural Network with two steps for face detection and classification. The images in the dataset is $7 * 18$ pixels, the images are in the gray scale in TIFF format from Yale face database. Images are preprocessed with purpose of reducing time and increasing the quality of image. In the first step, features are extracted from face image using Gabor filters with orientation of $5 * 8$ that is 40 filters. Second step is to classify the facial images obtained from feature vector using Neural network. The Neural network is trained with face and non-face images. The performance rate is $84.4 \%$.

\subsection{Empirical Background}

This section provides an overview of related work. Mood detection by facial image recognition is currently been used and tested in several fields for different reasons. As a result, a number of systems were developed that are specialized in mood detection by using facial recognition.

\subsubsection{Mood Detection in Recruitment}

In business world, this concept has grown up from project research to applicable systems. It becomes very essential for the companies to evaluate its scales. In business, scaling is the process of enabling the company's growth which require adapting new system, employees, technology in a cost-effective way which leads to overall efficiency and provide a competitive advantage. Many brands companies have started using mood detection technology not only to identify the consumer behavior, but also, to be used in recruitment process. Unilever company is applying facial emotions detections in hiring new employees by developing machine learning algorithm. The technology helps the company to select the right person for the right job. One of the process stages is the candidate send a video interview by answering the questions for 30 minutes by (Hire Vue) system. The system assesses the candidate confidence level based on facial expression and body language. By using natural language process, it becomes convenient in detecting their mood using their facial emotions and examine the personal traits required for specific job and make the decision if the candidate is suitable for the job. This technology helps to assess more than one million job applications per year. The average time of hiring process decreased from 4 months to 4 weeks, it also saved around 50000 hours of candidates' time. The time spent to review the job applications was decreased by $75 \%[14,22]$.

\subsubsection{Market Research}

Another application is Disney company research team developed a technology called FVAE or "Factorized Variational Autoencoders. The system is using facial recognition to predict the audience reaction while watching the movie. The company is using deep learning to detect audience facial expressions to assess their emotions during the movie time. The algorithm analyzes facial expression of the member of audience for 10 minutes and predict how the reaction will be to the rest of a film. FVAEs were able to learn some emotions like smile, laugh. Disney team used four infrared cameras and filmed the audience in 400 seats in theatre during 150 shows of 9 movies. Till now dataset of 16 million facial features of 3,179 audience member which manipulated by neural network have been stored in the database. FVAEs translated the datapoint into a series number denote the facial features (see figure 1). These numbers correlated to other data in metadata and allow the system to detect the audience reaction during watching the movie after just a few minutes like how much face is laughing and how much wide-open eye area is visible [15, 16, 24].

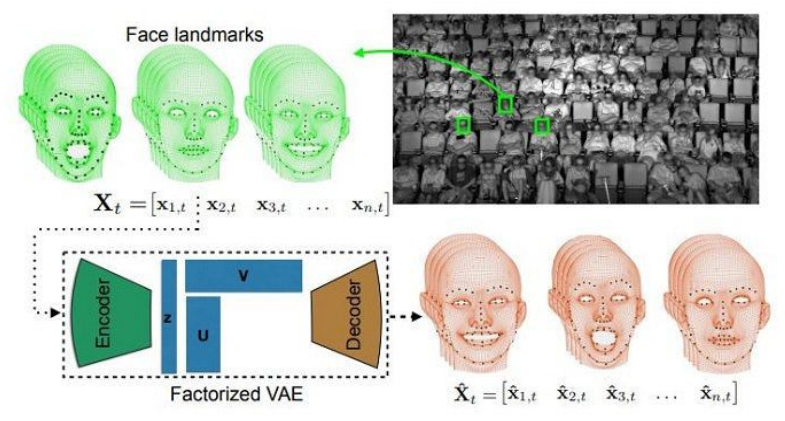

Figure 1: Facial Recognition in using FVAE technology in Disney

\subsubsection{Mood Detection in Retail Companies}

Westfield mall used a smart screen scan system developed by French software firm Quividi in the year 2015. Across 41 Westfield malls in different places in Australia and New Zealand, the shoppers scanned by hidden cameras in more than 1,600 billboards installed in the malls near the gates as show in figure 2 . The hidden cameras are not just used to recognize the shoppers age and gender but also to detect the mood. Hidden cameras captured blurry images of shoppers and processed by statistical analysis to identify shopper's mood and reaction towards brand and how they feel in the store in certain time. The system can distinguish five emotions from happy to unhappy. Quividi said the mood is hard to detect, accuracy sitting around $80 \%$. The data are collected and shared with advertisers to understand the shopper's feelings toward particular brand [17, 23, 27].

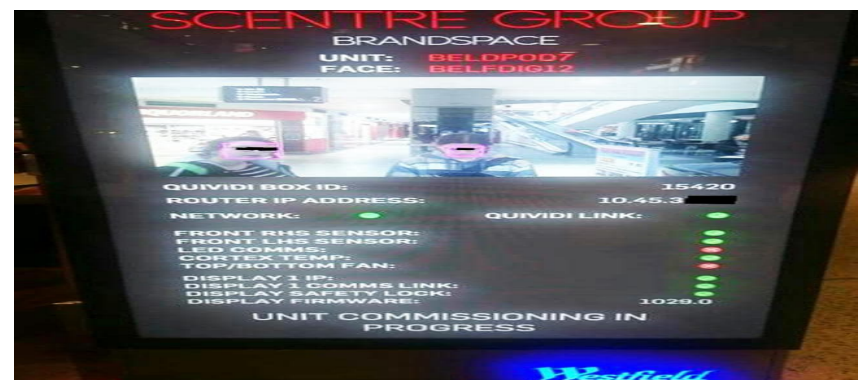

Figure 2:Billboard used for Mood Detection in Westfiled Mall 
As the literature above is critically analyzed; it is found that there have been few gaps observed in the existing literature. Gaps in literature can be clearly seen related to no study undertaken in terms of analyzing University students' subject areas of interest using facial recognition. Also, in this experiment some of the participants were female who wore a scarf and this is the first time that female with scarfs images included in this type of experiments. Gaps pertaining to test of various algorithm in machine learning is also seen and covering datasets and labelling them for its applications in various industries.

\section{METhODOLOGY}

The proposed methodology goes well in identifying how machine learning based on particular algorithm can be used to recognize person mood through his/her facial movement. This is a primary research based on experimental approach using quantitative analysis. The population sample taken for this research includes 15 random students seeking admission in ADSM. The facial mood recognition technology is being used by collecting images from cameras placed at ONE angle. A total of 60 independent variables have been identified in analyzing the images of facial expression during the whole experiment done in real time. The 15 participants include both gender and different age ranging from 10 to 40 years old. The demographic of those participants $53.4 \%$ are female, $46.6 \%$ are male, $93.33 \%$ are Emirati and 6.66\% Asian. Some of the participants were female who wore a scarf. The input images were taken by smart phone camera. The smart phone camera was placed in front of the person directly. The person performed four different facial expressions. The expressions based on four different expression which includes happy, sad, surprised and no emotion shown will be classified. These four emotions were used to help in understanding the psychological behavior and forms the basis of understanding their emotions related to selection of a particular program. Since the images size was large, we preprocessed and resized into 580 by 773 pixels array by using paint application.

The selected trained model was used to test input images to predict the emotions in different persons. As mentioned above, to overcome the inability to implement the proposed architecture system, various multiple trained models were deployed that took a long time to find the simple one to implement while giving a good emotion label probability. For deeper understanding to this study, a deep learning model that is developed by Kumar in [18] was used. Picking a right model that perform well with the problem is not an easy task but the reasons behind selecting this model are:

1. The dataset used to design the model is large, it contains 35,888 of 48 by 48 -pixel gray scale examples from Kaggle dataset [19].

2. The chosen model was trained using convolution neural network $(\mathrm{CNN})$ to classify the various expression into the algorithm which can be read emotions effectively for the images use as input because it does the feature engineering automatically while training and improve the model to yield what we demand,

3. The model gives $65-66 \%$ accuracy while training the model on validation set and for us this is considered to be better accuracy compared to other tested models which gives low accuracy,

4. Unlike the other tested models, this selected model while the codes are running it didn't produce any technical errors with our used pc system.

This trained model was selected initially in order to help to utilize seven emotions labels for each face image :("angry"(0), "disgust"(1), "scared"(2), "happy"(3), "sad"(4), "surprised"(5), "No Emotion"(6)). However, we focused in this study on four emotions: ("happy", "sad", "surprised", "No Emotion").

Figure 3 shows our methodology in determining the mood detection (recognition) task.

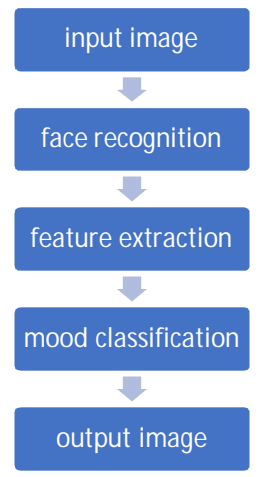

Figure 3: Mood Detection Methodology

The methodology includes input the student images for testing, apply face recognition task that recognizes each face elements (eyes, nose, forehead, mouth, and ears), then apply feature selection task that enable to extract each face feature, then mood classification will be done to detect each student mood for the aim of understanding his/her emotion through the academic advising process. This process will help the academic advisor to communicate well with the students seeking admission assistant.

To understand the methodology that we have adapted, Figures 4 and 5 clearly explain the algorithm used for the aim of emotions detection through facial recognition. This include neural network which is a subset of deep learning. The input data in the form of text, images and speech passes through different layers and each layer modifies the input values and then morphed it into something useful which can further be used as predictive model; and input of data can be tweaked for the output required. These neural networks automatically then feature engineer the input images and provide greater accuracy in emotion recognition. 


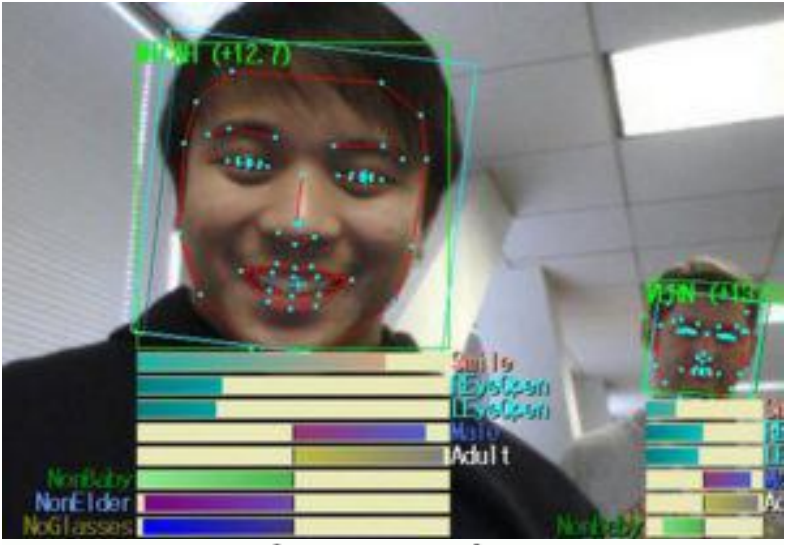

\section{Basic Emotions}

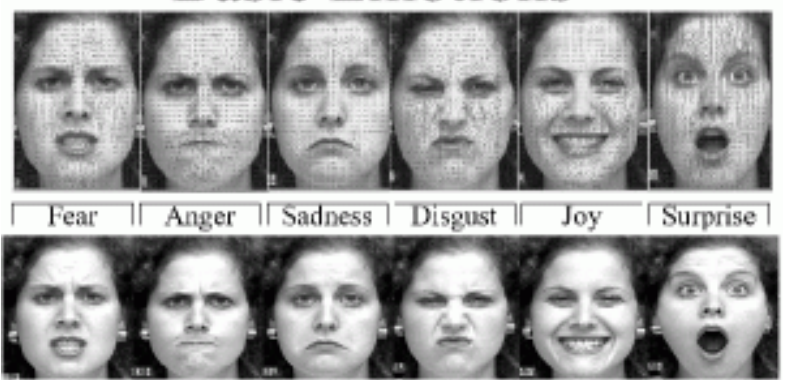

Figure 4:Detailed image. Source: (Algorthimia, 2018)

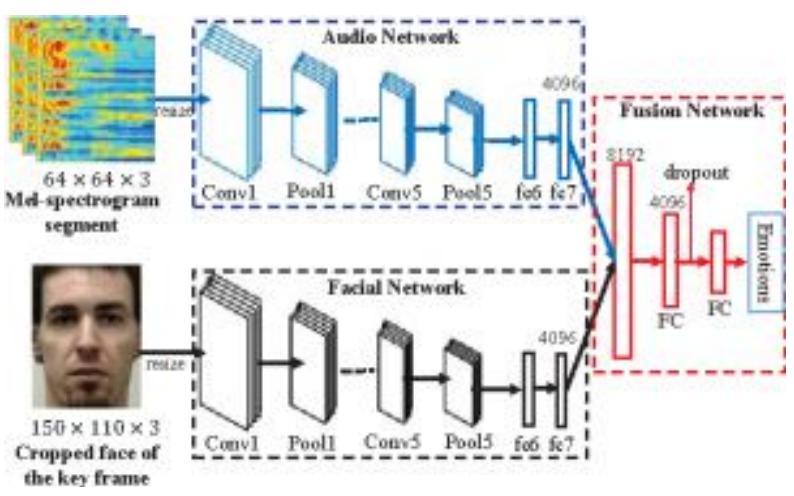

Figure 5: Neural network feature engineer for reading emotions. Source: (Algorthimia, 2018)

The original model comprised of two steps process. First the face detection of input image and then the emotion detection of bounded face in the input image. These steps are required to apply two techniques: first the developer was using Haar-feature cascade classifier which designed by open cv and used to detect an object from the image. This classifier is able to detect the frontal face of input image and in our study, it returned a red square bounding box coordinates. The face bounding box size is 48 by 48 pixel. Second technique, the developer was using Xception model which is a convolutional neural network that trained on more than a million image and can classify images into 1000 object categories so the network learned rich feature for a wide variety of images.
Subsequently the network takes the input image and then yield a label for the object in the image with the probabilities for each object of the categories [20].

According to the above, Xception can helps us to classify the face emotions into seven emotions with the given probabilities for each emotion, but as mentioned above we focused on four emotions: No Emotion, happy, sad and surprised. The output is detected emotions probabilities and emotions label on the left upper bounding box. It returned a matrix where each column represents the category and each row relates to one emotion probability. We tested our input images by implemented the codes in python 3.7 using keras by downloading it from anaconda. Keras is a useful library for developing deep learning models. It built on top of existing framework like tensorflow. It can be used for prediction and feature extraction that help us to achieve our study purpose. In order to run the codes, we rely on some libraries to execute the codes like: keras, open cv, numpy, imutils and tensorflow. The probabilities were calculated with emotion labels and inserted in a table which will be discussed in the next chapter. Furthermore after testing and analyzing the codes used in facial extraction, mood classification can be done and a clear output image will be identified

\section{Results AND Discussion}

The proposed research questions have been addressed and have been successfully answered through this experimental analysis. The research output was tested using 60 independent input colored images for 15 participants taken by smart phone camera in a pre-trained model. Through critically reviewing previous studies in the field of identifying the mood of people using facial recognition technology, the studies didn't address the values and habits of people, especially those related to the female whose wearing veil, which necessitated that we address this aspect to know the extent of the impact of machine learning on recognition on the mood of the person wearing the head scarf (veil), and the study found the mood detection using facial recognition technology give us the same results that the model can detect the mood of person with scarf or without it.

The model trained by using deep learning through convolution neural network. At first, it is important to understand that convolution neural network; it's one the most important concept in deep learning. It's a powerful neural network type that used in image classification in which can detect an object in an image. The main three components and layers are: convolutional layers, Max pooling layers and fully connected layers as output as showed below in figure 6. A convolution basically is a matrix called kernel filter passed over the input layer to create the feature map where extracting the features for the following layer. Each convolutional layer extract certain features. Convolutional layers have filters that each one slice the input image to extract meaningful information. Second type of layers is pooling layers, the main purpose of this type is to down sample the image because the deep learning is very complex, it has many of parameters and the computation is very time consumed so we need to down sample the image in each layer to have less parameters. For 
example, in figure 5 one of the most common pooling type is the max pooling which takes the maximum value from array of numbers. The third type is the fully connected layer in which one or two layers will be fully connected so the image will be converted to a vector of fully connected layer as the input layer[21].

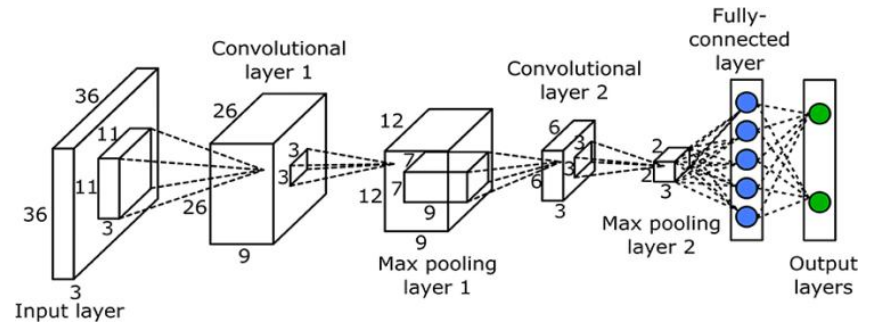

Figure 6:Architecture of deep convolution neural network with many layers

Hear feature cascade classifier is used to detect and extract the discriminative facial features like (eye, eyebrow, nose and mouth) and remove all unnecessary area of face and that step is a very important for facial emotions classification. Moreover, it reduces the time complexity in real time mood detection by facial recognition. In this experiment, we are successful in recognizing the facial in all tested input images and the output image is a red bounded box coordinate (see figure 7). At the second step, Xception classifier which we mentioned above(Section3) is used to classify the extracted emotion with given probabilities for each classified emotion and label it based on the highest-class probability. In this step we answered our first question: could machine learning deduce person mood from his/her facial movement? The model is capable to recognize the face and detect the emotion in seconds.

Table 1 shows the results of all tested input images. It can be seen that the model performed very well in classifying the happy emotions in 14 input images and 1 input image is misclassified and label it as No Emotion. The model performed a high probability ranging from 0.952843 to 0.997606 . Figure 8 shows how the facial movement is simple when the person is happy. The movement consists of rising cheeks, lip part and pulling the lip edges. Thus, these movements are very easy for the participants to represent it perfectly. No Emotion face is the emotion without facial movements and the model was successful in classifying most of the input images that the person was acting No Emotion (relaxing state without facial muscles movement) except 4 input images which classified as happy and this probably happen because the participants did minimal facial movement. The probability of classified emotion as No Emotion ranging from 0.438304 to 0.817396 . While the photos were taken, the process itself posed some challenges for the participants. It was found that some of facial expressions are difficult for the participants to represent especially the sad and surprise. Even though during the experiment the participants were subjected in a way to trigger their feelings, it was difficult for some of them to manipulate the sadness signs together such as: eye looking down with upper eyelids dropped, lip edges pulled down and inner corners of eyebrow pulled up together as show in figure 9, so that the images re-captured many times until the participants could represent the expression in a good way.

As shown in Table 1, it can be observed that the sad mood performed the worst among the four emotions that our goal to detect. From 15 participants, it was only possible to detect 2 of them in a sad mood label with a given probability of 0.536507 . On the other hand, the other 13 input images of people who performed a sad facial expression ,while during testing by the model, the model classified the input images as No Emotion label with probability fluctuating from 0.0293786 to 0.400591 . Although some of the participants struggled to represent the surprise facial expressions easily .The participant found it awkward and confused how to represent the surprise function as show in figure 10, which consists of inner brow rising, outer brow riser, upper lid riser and jaw drop, But when the input images tested, the model could classify 7 of them for the participants who represent the surprised facial expression and labeled them as surprise. The output probabilities are between 0.405041 to 0.798313 whereas the rest of input images classification varied between happy and scared labels.

Table 2 shows the details of classification results. Table 3 shows the confusion matrix that allow us to assess the performance of classification model. First row shows the predicted mood for every actual mood. It was noticed that the trained model suffers from poor discrimination for the sadness and surprised mood for the tested input images. While the trained model discriminates well on the happy mood, it results in mix the facial features and not to discriminate one mood from the other in sadness, No Emotion and surprised. Sadness mood has been classified as negative and few moods among surprise, no emotions have been classified as happy or positive. An example the whole experiment results can be seen in Figure 7 in which providing the facial and mood detection with visualizing each detected mood. The $\mathrm{x}$-axis in graphs represents the mood label while the y-axis represents the probability of detected mood. The graph for each mood shown that the model expected each mood and classified upon the highest given probability. The best performance for the classifier was detected the happy mood with high probability 0.996 , while the low performance in detecting the surprised mood and this is probably of participant mixed facial features between scared and no emotions that confused the classified. The research evaluated the performance of mood classification using the trained model in term of calculation the classification rate of correctly mood classified. The robustness of the trained model was tested by 60 independent input images where the classification rate of mood correctly classified was $56.6 \%$.

Table 1:Input Images Mood Classification with Probabilities

\begin{tabular}{|c|l|l|l|}
\hline participant & Input image & $\begin{array}{l}\text { Output } \\
\text { image }\end{array}$ & probabilities \\
\hline \multirow{3}{*}{1} & happy & happy & 0.992827 \\
\cline { 2 - 4 } & sad & No Emotion & 0.464419 \\
\cline { 2 - 4 } & No Emotion & No Emotion & 0.438304 \\
\hline
\end{tabular}




\begin{tabular}{|c|c|c|c|}
\hline & surprised & happy & 0.992147 \\
\hline \multirow{4}{*}{2} & happy & happy & 0.996849 \\
\hline & sad & No Emotion & 0.400591 \\
\hline & No Emotion & No Emotion & 0.71939 \\
\hline & surprised & & \\
\hline \multirow{4}{*}{3} & happy & happy & 0.997606 \\
\hline & sad & No Emotion & 0.536507 \\
\hline & No Emotion & No Emotion & 0.690962 \\
\hline & surprised & scared & 0.49667 \\
\hline \multirow{4}{*}{4} & happy & happy & 0.995016 \\
\hline & sad & No Emotion & 0.37942 \\
\hline & No Emotion & happy & 0.52818 \\
\hline & surprised & surprised & 0.739493 \\
\hline \multirow{4}{*}{5} & happy & happy & 0.996779 \\
\hline & sad & No Emotion & 0.561174 \\
\hline & No Emotion & No Emotion & 0.467919 \\
\hline & surprised & surprised & 0.798313 \\
\hline \multirow{4}{*}{6} & happy & happy & 0.996365 \\
\hline & $\mathrm{sad}$ & sad & 0.524801 \\
\hline & No Emotion & No Emotion & 0.473473 \\
\hline & surprised & surprised & 0.405041 \\
\hline \multirow{4}{*}{7} & happy & happy & 0.995592 \\
\hline & sad & No Emotion & 0.724729 \\
\hline & No Emotion & No Emotion & 0.817396 \\
\hline & surprised & happy & 0.725887 \\
\hline \multirow{4}{*}{8} & happy & happy & 0.964745 \\
\hline & sad & sad & 0.375585 \\
\hline & No Emotion & No Emotion & 0.7282249 \\
\hline & surprised & happy & 0.577858 \\
\hline \multirow{4}{*}{9} & happy & happy & 0.995908 \\
\hline & sad & No Emotion & 0.455605 \\
\hline & No Emotion & No Emotion & 0.495422 \\
\hline & surprised & happy & 0.714999 \\
\hline \multirow{4}{*}{10} & happy & happy & 0.952843 \\
\hline & sad & angry & 0.3522448 \\
\hline & No Emotion & No Emotion & 0.757347 \\
\hline & surprised & surprised & 0.519908 \\
\hline \multirow{4}{*}{11} & happy & happy & 0.994616 \\
\hline & sad & No Emotion & 0.7579265 \\
\hline & No Emotion & No Emotion & 0.757927 \\
\hline & surprised & happy & 0.474471 \\
\hline \multirow{4}{*}{12} & happy & happy & 0.984151 \\
\hline & sad & No Emotion & 0.584271 \\
\hline & No Emotion & happy & 0.877837 \\
\hline & surprised & happy & 0.568499 \\
\hline \multirow{4}{*}{13} & happy & No Emotion & 0.548583 \\
\hline & sad & No Emotion & 0.915304 \\
\hline & No Emotion & happy & 0.958487 \\
\hline & surprised & happy & 0.958543 \\
\hline \multirow{4}{*}{14} & happy & happy & 0.983672 \\
\hline & sad & No Emotion & 0.586976 \\
\hline & No Emotion & No Emotion & 0.708185 \\
\hline & surprised & surprised & 0.482892 \\
\hline \multirow{4}{*}{15} & happy & No Emotion & 0.385676 \\
\hline & sad & No Emotion & 0.289165 \\
\hline & No Emotion & happy & 0.419842 \\
\hline & surprised & happy & 0.988668 \\
\hline
\end{tabular}

Table 2:Mood Classification Results.

\begin{tabular}{|c|c|c|c|c|}
\hline \multirow{2}{*}{$\begin{array}{c}\text { Actual } \\
\text { class }\end{array}$} & \multicolumn{4}{|c|}{ Predicted class } \\
\cline { 2 - 5 } & happy & sad & $\begin{array}{c}\text { No } \\
\text { Emotion }\end{array}$ & surprised \\
\hline happy & 14 & 0 & 1 & 0 \\
\hline sad & 0 & 2 & 12 & 0 \\
\hline $\begin{array}{c}\text { No } \\
\text { Emotion }\end{array}$ & 4 & 0 & 11 & 0 \\
\hline surprised & 7 & 0 & 1 & 7 \\
\hline
\end{tabular}

Table 3:Confusion Matrix of Classification of Tested Input Data.

\begin{tabular}{|c|c|c|c|}
\hline Emotions & $\begin{array}{c}\text { Correctly } \\
\text { classified }\end{array}$ & $\begin{array}{c}\text { Wrong } \\
\text { classified }\end{array}$ & total \\
\hline Happy & 14 & 1 & 15 \\
\hline Sad & 2 & 13 & 15 \\
\hline No Emotions & 10 & 5 & 15 \\
\hline surprised & 7 & 8 & 15 \\
\hline Total & 34 & 26 & 60 \\
\hline $\begin{array}{c}\text { Classification } \\
\text { Rate }\end{array}$ & $56.6 \%$ \\
\hline
\end{tabular}

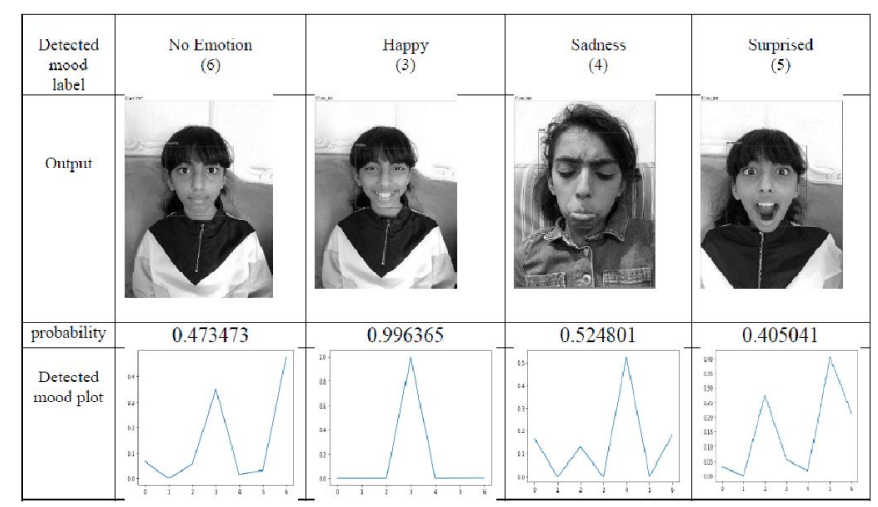

Figure 7:Example of the output tested image

Subsequent to the results discussed above, the research undertaken was successful in recognizing the facial of female with scarf and the model could detect their emotions. Happy and population with no emotion performed the best. While the sadness performed the worst. Furthermore, the surprised performed well. From Table 1, it was noted that even though the experiment couldn't correctly classify some input images but by looking to the probabilities of incorrectly classified input images, it either the second or third maximum probability and in some example were closer to the correctly classify probability. Based on the results analysis, the research seeks to highlight that some expressions represent from western people like sadness are different than Arabs representing and those people from Asia, since the model were trained by using western images dataset while we used Arab origin participants input images and compared it from students of Asian origin. Indeed, the performance of detecting 
the sadness mood was the worst. Moreover, the features the research took in account when the pictures were taken of the participants, they might be confused by the Xception classifier while test and analysis was performed through the input images; the features might be different than the dataset used to train the original model and for this reason such difference is found. On this basis the research emphasizes that understanding the facial expressions based on culture should also be consider when building a model or trying pre trained model. The findings clearly infer that machine learning and deep learning have the ability to recognize the human face and detect human mood by extraction the facial features. From this experiment, despite using a pre trained model with small number of individual input images to test them, the experiment was able to classify $56.6 \%$ of those input images correctly. The experiment was able to detect the four mood that we are interested in learning in order to achieve the goal of the proposed system. Knowing student mood is very important in communication. Understanding the student mood will help a lot in facilitating the communication between him/her and the academic advisor. This will further help the advisor to asset the student by giving him/her a proper information about the majors and provided by statistics information and facts and at the same time all these things will also help the student to at least make the right decision about appropriate major and eliminate the waste time. By testing the trained model, it was confirmed that machine learning and deep learning are very helpful in academic advising.

Here in this research trained model was used through convolution neural network approach to extract the facial features and classify the emotions. Due to lack of time, it was not possible to design an outwardly new model and it was not possible to take more images to test the model.

The findings of this study will certainly help in instigating future research on this realm and planning to extend this research. Future research can be taken forward from here and will help in building a new model by using another machine learning approach with using a large dataset that will try to detect seven emotions and develop a real time mood detection by facial recognition system. The research clearly indicates that there are factors which affect people emotions in real life which may cause them to change significantly either exposed to more or less to some extent.

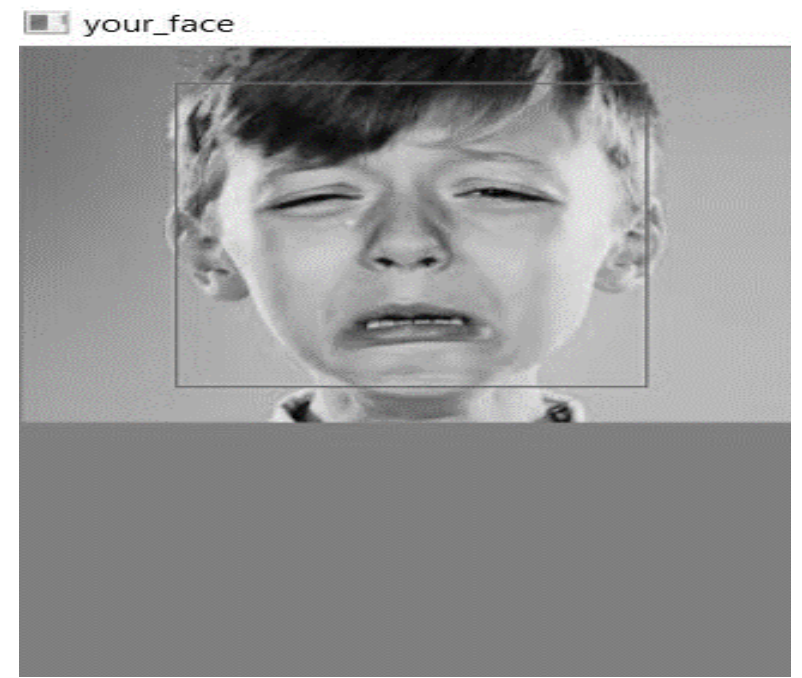

Figure 8:facial bounded box for tested image

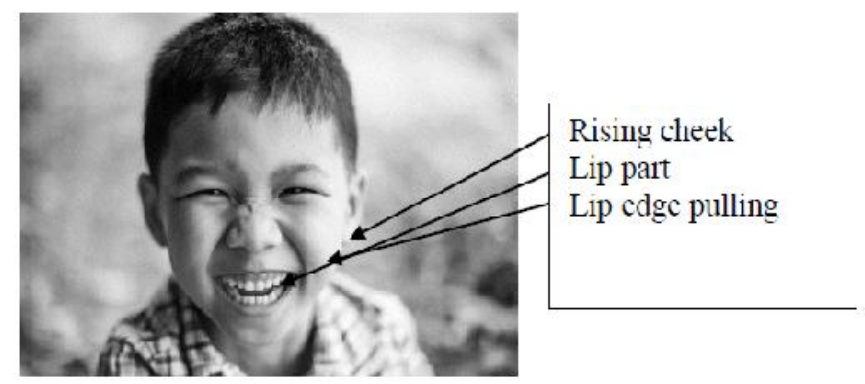

Figure 9:Human happy signs

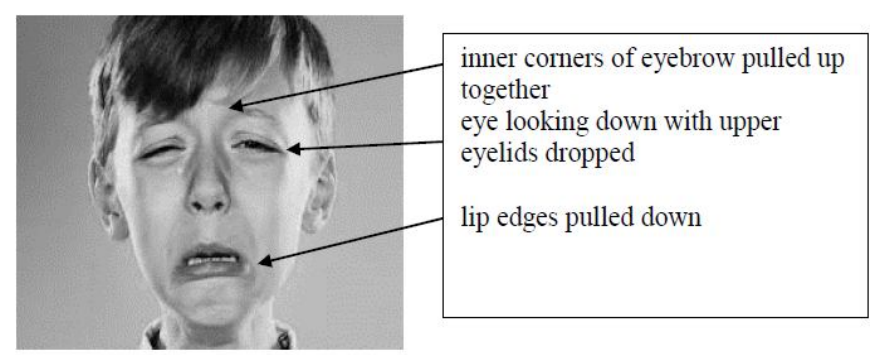

Figure 10:Human sadness signs 


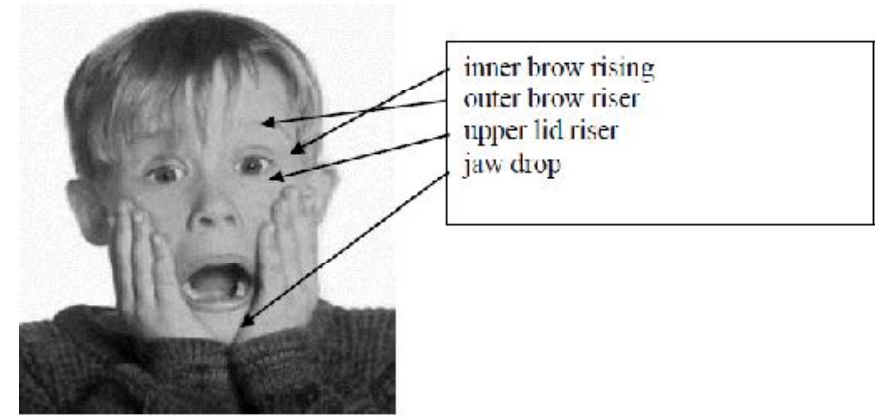

Figure 11:Human surprise signs

\section{CONCLUSION}

The study conducted clearly points out that mood detection using facial recognition technology has a lot of scope for improvement. The emotions such as happiness and no emotion were fairly easy to detect whereas the emotion which relates to sadness and surprised was not easy to detect. Moreover, expression and emotions expressed by different cultures namely, Arabs, Asians and Westerners are also difficult to analyze. The study encompasses all these and come up with great analysis and has a lot of promising future. This study can get better and prediction rate will improve with more and more data collected. The mood detection using facial recognition technology will work through Artificial Intelligence and will become more accurate as it will be used more and more. The more images are trained; the prediction accuracy will improve over a period of time. The current correct classification is $\mathbf{5 6 . 6}$ which is quiet good considering that only testing sample of 60 input images were taken. This is remarkable in the sense that in future work if the sample size would have been close to thousand the classification accuracy would have been higher, and it would have been much higher if the sample size would have been large. Ultimately this technology can reach good classification accuracy over a period of time as more and more data will be trained. The result of this research will be used in student's mood detection in academic advising process in ADSM for the aim of helping the students seeking academic assistant especially in choosing the right program to enroll in, it is suggested that this solution together with the solution provided by This solution will be improved for higher accuracy through training more data, and will be implemented to improve the academic advising and student requirement process in ADSM.

The recommendation provided on the basis of the study is that mood detection using facial recognition technology future is promising and can serve a large number of purposes. Also, privacy related matters need to be considered and manipulation of any kind should be avoided. It is also recommended that extra caution is required while reading the emotions as not emotions shown in the settings will be the outcome of the services offered and can be due to external forces as explained above. AI technology application will improve the whole process of predicting more accurate results.

\section{REFERENCES}

1. A Nelson \& Co. How do our emotions affect decision making? 2020. Retrieved from: https://www.bachremedies.com/en-us/emotional-he alth-blog/how-do-our-emotions-affect-decision-mak ing

2. A. Das, N. P. Reddy, and J. Narayanan. Hybrid fuzzy logic committee neural networks for recognition of swallow acceleration signals. Computer Methods and Programs in Biomedicine, vol. 64, no. 2, pp.87-99, 2001.

3. A. P. Gosavi, and S. R. Khot. Facial expression recognition using principal component analysis. International Journal of Soft Computing and Engineering (IJSCE), vol. 3, no.4, pp.2231-2307, 2013.

4. A. D. Kumar. Novel deep learning model for traffic sign detection using capsule networks. arXiv preprint arXiv:1805.04424.

5. A. Hirschkind, N., Mollick, S., Pari, M., \&Khim, J. M. (2020) Convolution neural network. Retrieved from

brillient.org:https://brilliant.org/wiki/convolutionalneural-network/

6. Algorithmia. Introduction to facial emotion recognition. 2018. Retrieved from: https://algorithmia.com/blog/introduction-to-emotio n-recognition

7. A. Hussien, M. Al-Kafri, A.A. Abonamah, M.U. Tariq (2020). Mood detection based Arabic text documents using machine learning methods, International Journal of Advanced Trends in Computer Science and Engineering, 9(4), 4224-4336.

8. B.M. Saleh, R.A. Al-besher, M.U. Tariq (2020). D-Talk: Sing Language Recognition System for People with Disability using Machine Learning and Image Processing, International Journal of Advanced Trends in Computer Science and Engineering, 9(4), 4374-4382.

9. E. Gillespie. Are you being scanned? How facial recognition technology follows you, even as you shop. The Guardian, February 24, 2019. Retrieved from:

https://www.theguardian.com/technology/2019/feb/ 24/are-you-being-scanned-how-facial-recognition-te chnology-follows-you-even-as-you-shop

10. G. Hemalatha, and C. P. Sumathi. A study of techniques for facial detection and expression classification. International Journal of Computer Science and Engineering Survey, vol. 5, no.2, pp. 27-37, 2014.

11. G. Mezzofiore. Disney is using facial recognition to predict how you'll react to movies. Mashable, July 17, 2017. Retrieved from: https://mashable.com/2017/07/27/disney-facial-reco gnition-prediction-movies/ 
12. Kulkarni, S. S. Kulkarni, N. P. Reddy, and S. I. Hariharan. Facial expression (mood) recognition from facial images using committee neural networks. Biomedical Engineering Online, 8(1), p. 16, 2009.

13. Lucey, P., Cohn, J. F., Kanade, T., Saragih, J., Ambadar, Z., \& Matthews, I. (2010, June). The extended cohn-kanade dataset $(\mathrm{ck}+)$ : A complete dataset for action unit and emotion-specified expression. In 2010 ieee computer society conference on computer vision and pattern recognition-workshops (pp. 94-101). IEEE.

14. L. F Barrett, R. Adolphs, S. Marsella, A. M. Martinez, and S. D. Pollak. Emotional expressions reconsidered: challenges to inferring emotion from human facial movements, Psychological Science in the Public Interest, vol. 20, no. 1, pp. 1-68, 2019.

15. L. F. Chen, H. Y. M. Liao, M. T. Ko, J. C. Lin, and G. J. Yu. A new LDA-based face recognition system which can solve the small sample size problem. Pattern Recognition, vol. 33, no. 10, pp. 1713-1726, 2000.

16. M. Verma, P. Rani, and H. Kundra. A hybrid approach to human face detection. International Journal of Computer Applications, vol.1, no. 13, pp.67-69, 2010.

17. M. Babar, A.S. Khattak, F.Arif, M.U. Tariq (2020). An improved framework for modelling data warehouse systems using UML Profile, International Arab Journal of Information Technology, 17(4). 562-570

18. M.U. Tariq, M.B. Bashir, M. Babar, A. Sohail (2020). Code Readability Management of High-Level Programming Languages: A Comparative Study, International Journal of Advanced Computer Science and Applications, 11(3), 595-602

19. M. Babar, M.U. Tariq, M.A. Jan (2020). Secure and Resilient Demand Side Management Engine using Machine Learning for IoT-enabled Smart Grid, Sustainable Cities and Societies, 62: 102370

20. N. P. Reddy, and O. A. Buch. Speaker verification using committee neural networks. Computer Methods and Programs in Biomedicine, vol. 72, no. 2, pp. 109-115, 2009.

21. N. A. Shrirao, N. P. Reddy, and Kosuri, D. R. Neural network committees for finger joint angle estimation from surface EMG signals. Biomedical Engineering Online, vol. 8, no.1, pp.2, 2009.

22. N. Gilliland. How brands are using emotion-detection technology. 2018 Retrieved from: https://econsultancy.com/how-brands-are-using-em otion-detection-technology/.

23. S. Aqab, M.U. Tariq (2020). Handwriting Recognition using Artificial Intelligence Neural Network and Image Processing, International Journal of Advanced Computer Science and Applications, 11(7).

24. S. Gupta. Facial emotion detection using AI: Use-cases. $2018 . \quad$ Retrieved from: https://hackernoon.com/facial-emotion-detection-usi ng-ai-use-cases-b7986bdc5700.

25. Y. Wang, H. Ai, B. Wu., and C. Huang. Real time facial expression recognition with adaboost., in Proceedings of the 17th International Conference on Pattern Recognition, 2004. ICPR August 2004. pp. 926-929.

26. Z. Niu, and X. Qiu. Facial expression recognition based on weighted principal component analysis and support vector machines, in 2010 3rd International Conference on Advanced Computer Theory and Engineering (ICACTE), August 2010, pp. V3-174.

27. Z. Hui, M. Babar, M.U. Tariq, M.A. Jan, V.G. Memon, X. Li (2020). SafeCity: Toward Safe and Secured Data Management Design for IoT-enabled Smart City Planning, IEEE Access, PP(99): 1-1 